\title{
Sustained behavioral recovery from unilateral nigrostriatal damage produced by the controlled release of dopamine from a silicone polymer pellet placed into the denervated striatum
}

\author{
Jill B. Becker ${ }^{1}$, Terry E. Robinson ${ }^{1}$, Peter Barton ${ }^{1}$, Ammon Sintov $^{2}$, Rivka Siden ${ }^{2}$ and \\ Robert J. Levy ${ }^{2}$ \\ ${ }^{\prime}$ Department of Psychology and Neuroscience Program, The University of Michigan, Ann Arbor, MI (U.S.A.) and ${ }^{2}$ Division of Pediatric \\ Cardiology, C.S. Mott Children's Hospital, The University of Michigan Medical Center, Ann Arbor, MI (U.S.A.)
}

(Accepted 27 June 1989)

Key words: Dopamine; Parkinson's disease; Controlled release; Rotational behavior; Apomorphine

\begin{abstract}
This study was conducted to determine if the behavioral asymmetry associated with unilateral nigrostriatal dopamine (DA) depletion could be alleviated by placing a small DA-releasing silicone polymer matrix pellet into the denervated striatum of rats. Animals that received DA-releasing pellets showed a 50\% reduction in apomorphine-induced rotational behavior, and this effect persisted for the 2-month duration of the experiment. The results suggest that the controlled release of DA from an intrastriatal polymer matrix can produce a long-lasting reduction in some of the symptons associated with DA depletion.
\end{abstract}

\section{INTRODUCTION}

Rats with a large unilateral lesion of the nigrostriatal dopamine (DA) system show behavioral asymmetries similar to those seen in people with hemi-Parkinsonism, and these behavioral asymmetries are accentuated by treatment with DA agonists ${ }^{18,19}$. For example, the directacting DA receptor agonist, apomorphine (APO), causes animals to turn in circles contralateral to the lesion side due to the action of APO at supersensitive DA receptors in the denervated striatum ${ }^{4,18,19}$. Grafts of DA-containing tissue (either fetal substantia nigra or adrenal medulla tissue) in or near the denervated striatum reduce the DA receptor supersensitivity, an effect that is manifest as a decrease in APO-induced turning behavior $6,7,9-11,14$.

The mechanism through which tissue grafts induce their behavioral effect is still unknown. One possibility is that tissue grafts alleviate the behavioral asymmetry produced by unilateral DA depletion by simply providing a supplementary source of DA. If this is the case, it may be possible to produce a similar restoration of function by providing the striatum with DA through alternative means.

The present experiment was designed as a preliminary test of this hypothesis. DA was provided via a small DA-silicone polymer pellet that slowly and continuously released DA. It is reported that placement of a DAreleasing pellet into the denervated striatum of rats with a unilateral nigrostriatal DA lesion reduced APO-evoked rotational behavior by $50 \%$ for the 2 -month duration of the experiment.

\section{MATERIALS AND METHODS}

Intrastriatal DA-silicone matrix implants

The DA-silicone matrix was prepared by mixing 20 parts of DA hydrochloride in 80 parts of polymethylsiloxane monomer (Silastic 382, Dow Corning, Midland, MI) with stannous octanoate as catalyst, using procedures similar to those previously described $^{12}$. The mixture was molded into strips $1 \mathrm{~cm}$ wide and $1 \mathrm{~mm}$ thick and cured at room temperature. Control implant material was prepared in the same way, but did not contain DA. Pellets for implantation were made by punching $1-\mathrm{mm}$ diameter pieces from the polymer strip with a stainless-steel punch, and then incubating the pellets for $18-24 \mathrm{~h}$ in $0.9 \%$ saline at $37^{\circ} \mathrm{C}$. Individual pellets weighed $0.64 \pm 0.05 \mathrm{mg}$ prior to incubation. Some pellets were placed into the striatum in one piece, and others were cut in half and placed at two sites within the striatum, as described below.

$D A$ release from silicone matrix implants

In vitro experiments were conducted to estimate the rate of DA release from the silicone polymer matrix. Pellets of DA-containing polymer $1 \mathrm{~mm}$ diameter $(n=5)$ were incubated in $0.05 \mathrm{M}$ potassium phosphate buffer with $10 \mathrm{mM} \mathrm{Na}_{2} \mathrm{~S}_{2} \mathrm{O}_{5}, \mathrm{pH} 7.4$ at $37^{\circ} \mathrm{C}$. Incubation was conducted in $1.5 \mathrm{ml}$ plastic-capped tubes. When these tubes were completely filled with buffer and tightly capped, no DA oxidation was observed. The incubation solutions were changed at: $1,2,3,6,7,10,14,21,28,35,42$ and 56 days. The solubilized DA released from the pellets was monitored by HPLC-EC as previously 
described $^{16}$ and the rate of DA release from the matrix was determined over the 7-week time period. In separate experiments, $1 \mathrm{~cm}^{2}$ pieces $(n=4)$ were incubated in $9 \mathrm{ml}$ buffer. DA release from the matrix in these experiments was quantified by absorbance at 279 $\mathrm{nm}$ at $1,2,3,6$ and $24 \mathrm{~h}$ and at $2,3,6,7,10,14,21,35$ and 42 days of incubation.

\section{Subjects and experimental procedure}

Male Holtzman rats (Madison, WI; $n=23$ ) weighing 200-250 g received a unilateral 6-hydroxydopamine lesion of the substantia nigra, as previously described ${ }^{15.10}$. Beginning 2 weeks after the lesion all animals were tested for baseline rates of APO-induced rotational behavior $(0.25 \mathrm{mg} / \mathrm{kg}$. s.c. $)$ in automated rotometers ${ }^{15}$. Each rat was tested $2-3$ times, with 3 days between tests. Only rats that made more than 90 full rotations $\left(360^{\circ}\right.$ turns) during at least two of the 40-min test sessions were included in the study. Animals that meet this criteria invariably have a $>90 \%$ striatal DA depletion.

Ten days after the last baseline test each animal was anesthetized with sodium pentobarbital supplemented with methoxyflurane. Using standard stereotaxic techniques and aseptic surgical procedures, one or two small pellets of the silicone-polymer matrix were placed into the denervated striatum of each animal using a trocar device constructed in the laboratory (coordinates (from Bregma, with Bregma and Lambda horizontal), single pellet: AP $+0.5 \mathrm{~mm}$. L $2.8 \mathrm{~mm}$, V $6.0 \mathrm{~mm}$; two pellets: (a) AP $-0.26 \mathrm{~mm}, \mathrm{~L} 3.0 \mathrm{~mm}$. V $5.5 \mathrm{~mm}$; and (b) AP $+1.2 \mathrm{~mm}$, L $2.5 \mathrm{~mm}$, V $5.5 \mathrm{~mm}$ ). Following surgery the animals were returned to their home cages and left undisturbed until retested for APO-induced rotational behavior 1 $3,10,30$ and 60 days later.

At the completion of the behavioral experiment the animals were perfused with $0.9 \%$ saline followed by $10 \%$ formalin. The brain were removed and stored in 10\% formalin until frozen sections (40) $\mu \mathrm{m}$ thick) were obtained. The location of the pellets within the brain was determined by examination of Cresyl violet-stained sections. Four animals were excluded because their pellets were not in the intended location.

\section{RESULTS}

The rate of DA release from the silicone matrix in vitro was not dramatically influenced by the shape/size of the matrix. As can be seen in Fig. 1, with both configurations of the matrix there is a very rapid release of DA during the first $24 \mathrm{~h}$ in vitro. The DA-silicone polymer matrix pellets used in the behavioral experiments released approximately $373.6 \pm 20.0 \mathrm{nmol} \mathrm{DA} /$ pellet (mean \pm S.E.M.) or about $55 \%$ of the DA contained in the pellet during this period prior to implantation. Between days 1 and 10 in vitro, the pellets released an additonal $37 \%$ of the total DA. Between days 10 and 60, DA release continued to decrease. A total of $1.69 \pm 0.13 \mathrm{nmol} \mathrm{DA} /$ pellet or $2.5 \%$ was released during this period of time in vitro. Release of DA from the larger pieces of DA-silicone matrix was slightly less during the initial dynamic phase of DA release, but the profile of the time course is quite similar (Fig. 1). It must be stressed, however, that how well release rate in vitro approximates release in vivo is not known.

Both control and experimental animals showed a marked, but transient, reduction in APO-induced turning behavior 1-3 days after implantation of the polymer pellets. The reduction was significantly greater, however,

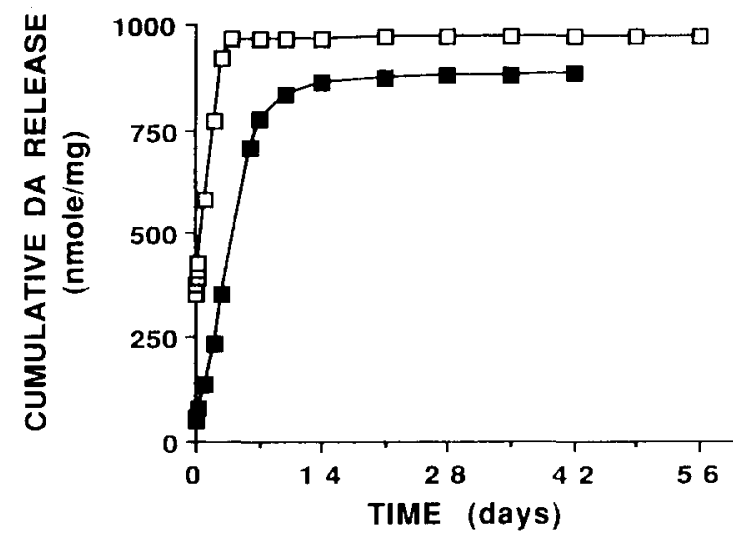

Fig. 1. Cumulative DA release (nmol DA/mg polymer) from $1-\mathrm{mm}$ diameter DA-silicone polymer matrix pellets (open squares; $n=5$ ) or $1 \mathrm{~cm}^{2}$ pieces (closed squares; $n=4$ ) during incubation in vitro (see text for additional details).

in animals with DA-releasing pellets. On day 3 postoperatively, turning in control animals was $77.3 \pm 9.5 \%$ (mean \pm S.E.M.) of baseline, whereas experimental animals turned at a rate that was only $32.0 \pm 8.1 \%$ of baseline (baseline $=100 \%$ ).

By 10 days postoperatively the rate of turning had stabilized in both experimental and control animals, but rats with DA-releasing pellets showed a greater reduction in turning behavior than did control animals for the remainder of the experiment $(P<0.002)$. Animals with

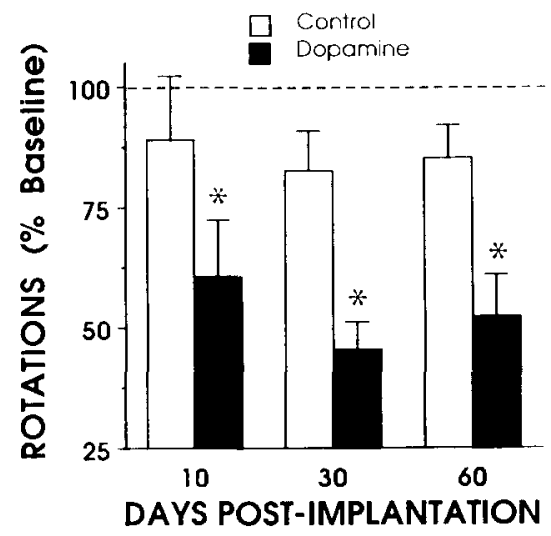

Fig. 2. Mean ( \pm S.E.M.) APO-induced rotational hehavior at 10 30 and 60) days postimplantation in control animals (open bars; $n=9$ ) and animals that received DA-releasing pellets (solid bars; $n=10)$ in the DA-denervated striatum. Rotational hehavior was induced by APO $(0.25 \mathrm{mg} / \mathrm{kg})$ and is expressed as a percent of the mean rate of turning prior to implantation of the silicone polymer pellets. Prior to implantation of the pellets animals made $253.4 \pm$ 31.6 full rotations/4)-min test session (mean \pm S.E.M.). The rate of turning 10-60 days postimplantation was significantly different from baseline in animals that received DA-releasing pellets $(P<0.007$, $t$-tests), but not in control animals. The asterisks indicate that animals with the DA-releasing pellets turned significantly less than did the control animals, as indicated by a significant main effect of group $\left(F_{1,17}=13.92 ; P<0.002\right)$ but no effect of time $(F=0.8)$ or group by time interaction $(F=0.1 ; 2$-way ANOVA with repeated measures 


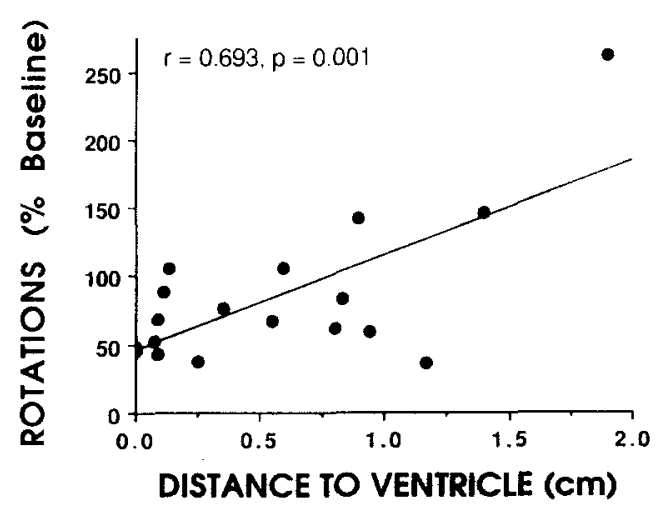

Fig. 3. Correlation between the distance from the ventricle of DA-releasing silicone polymer implants and the percent reduction in rotational behavior. Each data point represents one individual animal. Pellets placed close to the ventricle within the striatum were most effective in producing a reduction in APO-induced rotational behavior.

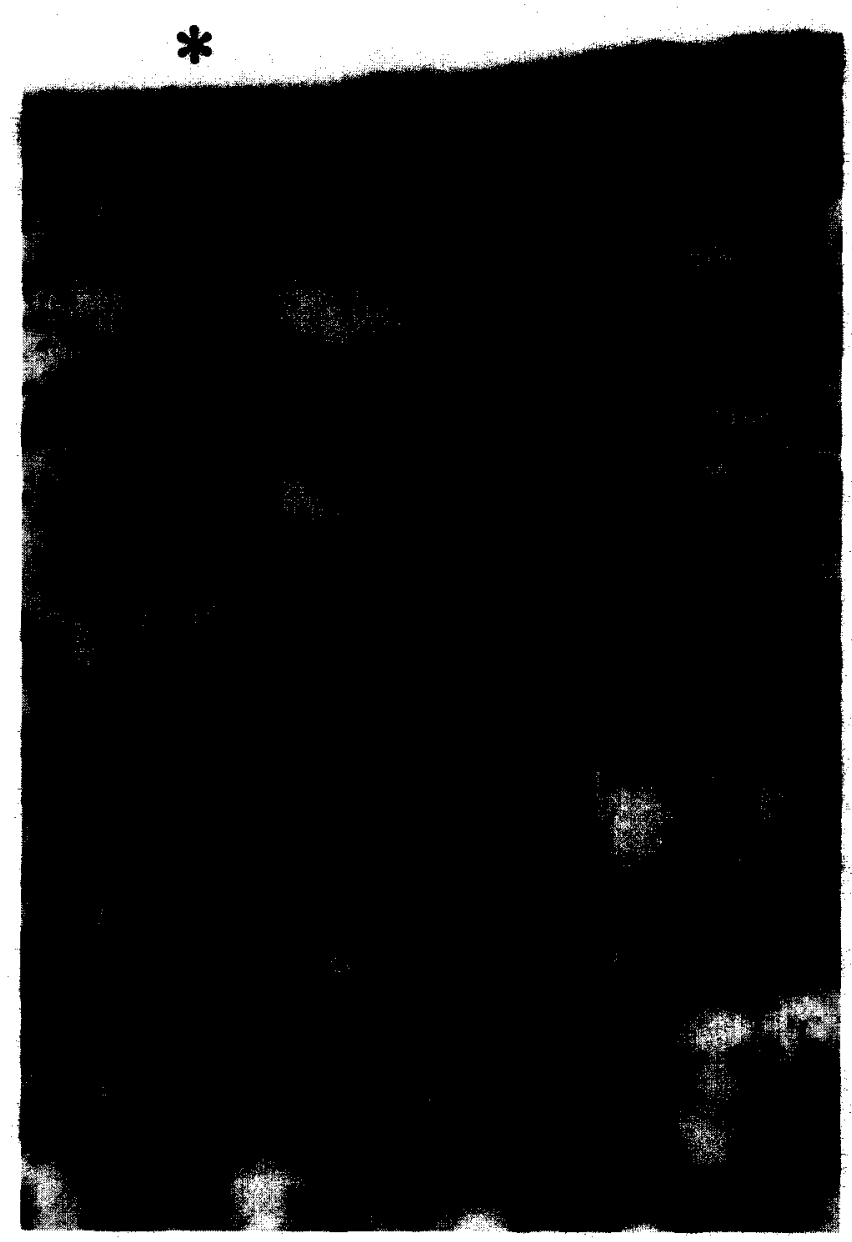

Fig. 4. Cresyl violet-stained section ( $40 \mu \mathrm{m}$ tick) of striatum adjacent to the site of a DA-silicone polymer pellet (initial negative enlargement $\times 50) .{ }^{*}$ Indicates the site where the pellet was found in the striatum. Note the thin layer of fibrous appearing material and gliosis immediately adjacent to the cavity formed by the pellet, but the normal appearance of the striatal neurons within a few microns of the implant site.
DA-silicone pellets turned at a rate that was less than $50 \%$ of baseline when animals were tested at 30 and 60 days after implantation (Fig. 2). In contrast, between 10 and 60 days postoperatively the rate of turning in control animals was not significantly different from baseline.

Interestingly, the location of the pellet within the striatum was a significant factor in how effectively the implant reduced rotational behavior (Fig. 3). The more lateral the placement the smaller the decrease in rotational behavior ( $r=0.693, P=0.001)$. This finding is similar to that described by Dunnett and colleagues using fetal substantia nigra grafts transplanted into striatum ${ }^{7.8}$. It should also be noted that in two animals the pellet was inadvertently positioned within the ventricle and in these animals rotational behavior was not reduced (data not shown).

Examination of the histology showed that there were no gross tissue compatibility problems with this polymer matrix. There was a thin layer of fibrous tissue and gliosis immediately adjacent to the site of implant, but normal appearing neurons within a few microns of the implant cavity (Fig. 4). This was expected because the silicone polymer has received FDA approval for biomedical applications. Similar tissue compatibility has been recently reported following implantation of polyvinyl acrylic copolymer capsules into neocortex ${ }^{1.20}$

\section{DISCUSSION}

Implantation of a silicone polymer pellet that released DA into the denervated striatum reduced the behavioral asymmetry associated with unilateral nigrostriatal DA depletion. While a transient reduction in rotational behavior was observed in control animals (probably due to non-specific trauma associated with the surgery) turning rates had returned to preimplant levels by day 10 . In contrast, animals with DA-releasing implants showed a significantly greater decrease in turning at 3 days postimplant, than did animals with control implants, and continued to exhibit reduced rotational behavior for 2 months. Exactly how long the pellets would continue to be effective in vivo remains to be determined.

The magnitude of the decrease in the APO-stimulated rotational behavior seen here is comparable to that obtained with other procedures. For example, a 25-50\% reduction in APO-induced rotation is seen with adrenal medulla grafts ${ }^{9,11,17}$, or with the intrastriatal infusion of DA $(0.5-5 \mu \mathrm{g} / \mathrm{h})$ using an osmotic mini-pump for two weeks (ref. 13; see also ref. 17). In the present study, in vitro release from the pellets decreased from 385 to 16 $\mathrm{pg} / \mathrm{h}$ (or from 150.8 to $6.3 \mathrm{fmol} / \mathrm{min}$ ) between days 10 and 56. Whereas this rate of DA release is very low, it is within the range of normal extracellular DA-release rates 
reported in microdialysis studies ${ }^{16}$ and the behavioral evidence indicates that release was sufficient to maintain suppression of rotational behavior by $50 \%$. It is also relevant that DA diffuses quite freely through a DAdepleted striatum, unlike the intact striatum where diffusion is prevented by re-uptake into presynaptic DA terminals ${ }^{5}$. It should be noted, that the rate of DA release during this time period was declining gradually and that in vitro experiments at best provide only a rough estimate of release rate in vivo. However, previous work by others has demonstrated that there is a close correspondence between in vitro and in vivo drug release with some polymer matrix systems ${ }^{3}$. Nevertheless, further experiments will be required to determine the in vivo rate of DA release from the type of silicone polymer pellets used here.

Although the magnitude of the reduction in APOinduced rotational behavior seen here was close to that seen with adrenal medulla grafts it is not known whether this represents the maximal effect possible using this procedure. It may be, for example, that the silicone polymer pellets were not optimally placed in the striatum for reducing rotational behavior, or that the dose was suboptimal. Whether other sites within the striatum may be more effective is currently under investigation, as are dose-response studies.

In conclusion, this preliminary study demonstrates that it is possible to produce a sustained reduction in the symptoms associated with striatal DA depletion by chronic implantation of an exogenous source of DA, such as the controlled release DA-silicone polymer matrix

\section{REFERENCES}

1 Aebischer, P., Winn, S.R. and Galletti, P.M., Transplantation of neural tissue in polymer capsules, Brain Research, 448 (1988) 364-368.

2 Björklund, A., Lindvall, O., Isacson, O., Brundin, P., Wictorin, K., Strecker, R.E., Clarke, D.J. and Dunnett, S.B., Mechanisms of action of intracerebral neural implants: studies on nigral and striatal grafts to the lesioned striatum, Trends Neurosci., 10 (1987) 509-516.

3 Brown, L.R., Wei, C.L. and Langer, R., In vivo and in vitro release of macromolecules from polymeric drug delivery systems, J. Pharmaceut. Sci., 72 (1983) 1181-1185.

4 Creese, I. and Snyder, S.H., Nigrostriatal lesions enhance striatal $\left[{ }^{3} \mathrm{H}\right]$ spiroperidol binding, Eur. J. Pharmacol., 56 (1979) 277-281.

5 Doucet, G., Descarries, L. and Garcia, S., Quantification of the dopamine innervation in adult rat neostriatum, Neuroscience, 19 (1986) $427-445$.

6 Dunnett, S.B., Björklund, A., Stenevi, U. and Iversen, S.D., CNS transplantation: structural and functional recovery from brain damage. In R.M. Buijs, P. Pevet aand D.F. Swaab (Eds.), Chemical Transmission in the Brain. Progress in Brain Research, Vol. 55, Elsevier, Amsterdam, 1982.

7 Dunnett, S.B., Björklund, A. and Stenevi, U., Transplantinduced recovery from brain lesions: a review of the nigrostriatal model. In R.B. Wallace and G.D. Das (Eds.), Neural Tissue pellet used here. This supports the idea that in some neural systems grafts may only need to provide a tonic, unregulated source of neurotransmitter to be effective; i.e. integration of the graft and host is not necessary ${ }^{2}$. Of course, many more experiments will be required to thoroughly evaluate the technique described here. For example, additional experiments will be required to determine the extent and rate of DA diffusion in vivo as well as the long-term stability of DA within the silicone polymer matrix. In addition, the polymer used was not ideal because release was continuously decreasing over time. Polymers from which release occurs at a constant rate (i.e. zero-order release kinetics) may be more beneficial. We are currently experimenting with the use of rate-limiting membranes ${ }^{12}$ to achieve zero-order release kinetics from polymer matrices of this type. Even more important to possible clinical applications, the ability of DA-releasing pellets to alleviate other behavioral symptoms of striatal DA depletion must be thoroughly evaluated (especially following bilateral insult). Nevertheless, the experiment reported here suggests this approach could potentially provide an alternative to tissue grafts in the treatment of Parkinson's Disease.

Acknowledgements. This research was supported by grants from the NIH: NS22157 to J.B.B., DA04294 to T.E.R. and HL38118 to R.J.L. J.B.B. and T.E.R. were supported by RCDAs: NS01056 and NS00844, respectively. We would like to thank the anonymous reviewer for the helpful comments made on an earlier version of the manuscript.

Transplantation Research, Springer, New York, 1983.

8 Dunnett, S.B., Björklund, A.. Stenevi, U. and Iversen, S.D., Grafts of embryonis substantia nigra reinervating the ventrolateral striatum ameliorate sensorimotor impairments and akinesia in rats with 6-OHDA lesions of the nigrostriatal pathway, Brain Research, 229 (1981) 209-217.

9 Freed, W.J., Functional brain tissue transplantation: reversal of lesion-induced rotation by intraventricular substantia nigra and adrenal medulla grafts, with a note on intracranial retinal grafts, Biol. Psychiat., 18 (1983) 1205-1267.

10 Freed, W.J., Ko, G.N., Niehoff, D.L., Kuhar, M.J., Hoffer, B.J., Olson, L., Cannon-Spoor, H.E.. Morihisa, J.M. and Wyatt, R.J., Normalization of spiroperidol binding in the denervated rat striatum by homologous grafts of substantia nigra, Science, 222 (1983) 937-939.

11 Freed, W.J., Morihisa, J.M., Spoor, H.E., Hoffer, B.J., Olson, L., Seiger, A. and Wyatt, R.J.. Transplanted adrenal chromaffin cells in rat brain to reduce lesion-induced rotational behavior. Nature (Lond.), 292 (1981) 351-352.

12 Golomb. G.. Dixon, M., Smith, M.S. . Schoen, F.J. and Levy, R.J., Controlled-release drug delivery of diphosphonates to inhibit bioprosthetic heart valve calcification: release rate modulation with silicone matrices via drug solubility and membrane coating, J. Pharmaceut. Sci., 76 (1987) 271-276.

13 Hargraves, R. and Freed, W.J., Chronic intrastriatal dopamine infusions in rats with unilateral lesions of the substantia nigra, Life Sci., 40 (1987) 959-966. 
14 Perlow, M.J., Freed, W.J., Hoffer, B.J.. Seiger, A., Olson, L. and Wyatt, R.J., Brain grafts reduce motor abnormalities produced by destruction of the nigrostriatal dopamine system, Science. 204 (1979) 547-643.

15 Robinson, T.E., Becker, J.B. and Presty, S.K., Long-term facilitation of amphetamine-induced rotational behavior and striatal dopamine release produced by a single exposure to amphetamine: sex differences, Brain Research, 253 (1982) 231-241.

16 Robinson, T.E. and Whishaw, I.Q., Normalization of extracellular dopamine in striatum following recovery from a partial unilateral 6-OHDA lesion of the substantia nigra: a microdialysis study in freely moving rats, Brain Research, 450 (1988) 209-224.

17 Strömberg, I.. Herrara-Marschitz, M., Ungerstedt, U. Ebendal.
T. ans Olson. L., Chronic implants of chromaftin tissue into the dopamine-denervated striatum. Effects of NGF on graft suvival. fiber growth and rotational behavior. Exp. Brain Res., 60 (1985) $335-349$.

18 Ungerstedt, U., Postsynaptic supersensitivity after thydroxydopamine induced degeneration of the nigrostriatal dopamine system, Acla Physiol. Scand., Suppl. 367, 82 (1971) 69-92.

19 Ungerstedt, U., Functional dynamics of central monoamine pathways. In F.O. Schmitt and F.G. Worden (Eds.). The Neurosciences: Third Study Program, MIT Press, Cambridge, MA, 1974, pp. 979-988.

20 Winn, S.R., Aebischer, P. and Galletti, P.M., Brain tissue reaction to permselective polymer capsules, J. Biomed. Mater. Res., 23 (1989) 31-44. 